E. Okassa

Nagoya Math. J.

Vol. 115 (1989), 63-71

\title{
RELÈVEMENTS DES STRUCTURES SYMPLECTIQUES ET PSEUDO-RIEMANNIENNES À DES VARIÉTÉS DE POINTS PROCHES
}

\author{
EUGÈNE OKASSA
}

On considère une variété différentielle $M$, paracompacte de classe $C^{\infty}$. Etant donné une algèbre locale $A$ (algèbre commutative unitaire de dimension finie sur $\mathbb{R}$ dont l'idéal maximal $m$ est de codimension 1 sur $\mathbb{R}$ ), on rappelle qu'un point proche de $x \in M$ d'espèce $A$ est un homomorphisme d'algèbres $\xi$ de $C^{\infty}(M)$ [algèbre des fonctions numériques de classe $C^{\infty}$ sur $M]$ dans $A$ tel que $\xi(f) \equiv f(x) \bmod m$ pour toute fonction $f \in C^{\infty}(M)$ [9]. En notant $M_{x}^{A}$ l'ensemble des points proches de $x$ d'espèce $A, M^{A}=\bigcup_{x \in M} M_{x}^{A}$ est une variété différentielle de dimension $n \times \operatorname{dim} A$ où $n=\operatorname{dim} M . \quad \mathrm{Si}$ $A=\mathbb{R}\left[T_{1}, \cdots, T_{s}\right] /\left(T_{1}, \cdots, T_{s}\right)^{k+1}$, la variété $M^{A}$ s'identifie à la variété des jets, $J_{0}^{k}\left(\mathbb{R}^{s}, M\right)$, des applications différentiables de classe $C^{\infty}$ de $\mathbb{R}^{s}$ dans $M$ ayant $0 \in \mathbb{R}^{s}$ pour source.

Si $f$ est une fonction de classe $C^{\infty}$ sur $M, f^{A}: M^{A} \rightarrow A$ est définie par $f^{A}(\xi)=\xi(f)$ pour tout $\xi \in M^{A}$.

\section{§1. Relèvement des formes différentielles}

On désigne par $\Omega(M)=\oplus_{p \in \mathbb{N}} \Omega^{p}(M)$ le $C^{\infty}(M)$-module gradué des formes différentielles sur $M$ et $\mathfrak{X}(M)$ le $C^{\infty}(M)$-module des champs de vecteurs sur $M$.

Proposition 1. Etant donné une forme différentielle $\omega$ de degré $p$ sur $M$, il existe une forme différentielle de degré $p$ et une seule $\omega^{A}$ sur $M^{A} \grave{a}$ valeurs dans A telle que

$$
\omega^{A}\left(a_{1} X_{1}^{A}, a_{2} X_{2}^{A}, \cdots, a_{p} X_{p}^{A}\right)=a_{1} \cdots a_{p}\left[\omega\left(X_{1}, \cdots, X_{p}\right)\right]^{A}
$$

pour tous $a_{1}, \cdots, a_{p}$ dans $A$ et $X_{1}, \cdots, X_{p}$ dans $\mathfrak{X}(M) ; X^{A}$ désigne le prolongement $\grave{a} M^{A} d u$ champ de vecteurs $X$ sur $M$.

Démonstration. En chaque point $\xi \in M^{A}$, les $a \cdot X^{A}(\xi)$, où $a \in A$ et Received March 29, 1988. 
$X \in \mathfrak{X}(M)$, engendrent l'espace $T_{\xi} M^{A}$. Il s'ensuit donc que $\omega^{A}$ est unique. Quant à l'existence, elle est immédiate.

Lorsque $\varphi \in A^{*}$ est une forme linéaire sur $A$ et $\omega$ une forme différentielle sur $M$, on dira que la forme scalaire $\varphi \circ \omega^{A}$ est une relevée de $\omega$ à $M^{A}$. Si $\omega$ est une forme différentielle de degré $p$ sur $M$, si $\left(F_{\alpha}\right)_{\alpha \in I}$ est une base de $A$ et $\left(F_{\alpha}^{*}\right)_{\alpha \in I}$ la base duale, on $a$ :

$$
\left(\varphi \circ \omega^{A}\right)\left(a_{1} X_{1}^{A}, a_{2} X_{2}^{A}, \cdots, a_{p} X_{p}^{A}\right)=\sum_{\alpha \in I} \varphi\left(a_{1} \cdots a_{p} F_{\alpha}\right) F_{\alpha}^{*} \circ\left[\omega\left(X_{1}, \cdots, X_{p}\right)\right]^{A} .
$$

Exemple. $\quad M=\mathbb{R}^{n} ; \quad A=\mathbb{D}$ l'algèbre des nombres duaux. Soit $\left(x_{1}, \cdots, x_{n}\right)$ le système de coordonnées canonique sur $\mathbb{R}^{n},\left(y_{1}, \cdots, y_{n}\right)$ les coordonnées sur la fibre de $M^{\mathrm{D}}=T \mathbb{R}=\mathbb{R}^{2 n}$. Soit $(1, \varepsilon)$ une base de $\mathbb{D}$ avec $\varepsilon^{2}=0$ et $\left(1^{*}, \varepsilon^{*}\right)$ la base duale. Si $\omega=\sum_{i=1} P_{i} d x_{i}$ est une forme différentielle de degré 1 sur $\mathbb{R}^{n}$, alors on $a$;

$$
\begin{aligned}
\omega^{\mathrm{D}} & =1 \otimes\left(\sum_{i=1}^{n} P_{i} d x_{i}\right)+\varepsilon \otimes\left[\sum_{i=1}^{n}\left(\sum_{j=1}^{n} y_{j} \frac{\partial P_{i}}{\partial x_{j}}\right) d x_{i}+\sum_{i=1}^{n} P_{i} d y_{i}\right] \\
1^{*} \circ \omega^{\mathrm{D}} & =\sum_{i=1}^{n} P_{i} d x_{i} \\
\varepsilon^{*} \circ \omega^{\mathrm{D}} & =\sum_{i=1}^{n}\left(\sum_{j=1}^{n} y_{j} \frac{\partial P_{i}}{\partial x_{j}}\right) d x_{i}+\sum_{i=1}^{n} P_{i} d y_{i}
\end{aligned}
$$

\section{Propriétés.}

i) $\left(\omega_{1}+\omega_{2}\right)^{A}=\omega_{1}^{A}+\omega_{2}^{A}$ pour tous $\omega_{1}$ et $\omega_{2}$ dans $\Omega(M)$

ii) $\left(\omega_{1} \wedge \omega_{2}\right)^{A}=\omega_{1}^{A} \wedge \omega_{2}^{A}$ pour tous $\omega_{1}$ et $\omega_{2}$ dans $\Omega(M)$

iii) $d\left(\omega^{A}\right)=(d \omega)^{A}$ pour tout $\omega \in \Omega(M)$ : $d$ est l'opérateur de différentiation extérieure.

iv) Pour tout champ de vecteurs $X$ sur $M$, pour tout $\omega \in \Omega(M)$, et pour tout $a \in A$, on a: $\theta_{a X^{A}}\left(\omega^{A}\right)=a\left(\theta_{X} \omega\right)^{A}$ où $\theta_{X}$ est la dérivée de Lie |par rapport au champ de vecteurs $X$.

v) Pour toute dérivation $\delta$ de $A$ et pour tout $\omega \in \Omega(M)$, on a: $\theta_{\delta_{M}} \omega^{A}=-\delta \circ \omega^{A}$ où $\delta_{M}$ est le champ de vecteurs sur $M^{A}$ associé à la dérivation $\delta$ [5].

On désigne par $\mu_{A}: A \times A \rightarrow A$ la multiplication dans $A$.

Proposition 2. Soit $(M, \omega)$ une variété symplectique. Etant donné $\varphi \in A^{*}$, on $a \operatorname{rang}\left(\varphi \circ \omega^{A}\right)=\operatorname{rang}\left(\varphi \circ \mu_{A}\right) \times \operatorname{dim} M$.

Démonstration. En chaque point $\xi \in M^{A}$, montrons que le noyau de $\left(\varphi \circ \omega^{A}\right)(\xi)$ est $\operatorname{ker}\left(\varphi \circ \mu_{A}\right) \cdot T_{\xi} M^{A}$. 
Soit $\xi$ un point proche de $x_{0} \in M$ d'espèce $A$. On suppose $\operatorname{dim} M=2 n$. Soit $\left(x_{1}, \cdots, x_{2 n}\right)$ un système de coordonnées locales dans un voisinage $U$ de $x_{0}$ tel que $\omega / U\left(\partial / \partial x_{i}, \partial / \partial x_{i+n}\right)=1$ pour tout $i=1,2, \cdots, n$ et $\omega / U\left(\partial / \partial x_{i}, \partial / \partial x_{j}\right)$ $=0$ pour $j \neq i+n$. On considère $\left(a_{\alpha}\right)_{\alpha \in I}$ une base de $A$ et $\left(a_{\alpha}^{*}\right)_{\alpha \in I}$ la base duale. Soit $X=\sum_{\substack{i=1,2, \ldots, 2 n \\ \alpha \in I}} \lambda_{i \alpha} a_{\alpha}\left(\partial / \partial x_{i}\right)^{A}(\xi)$ un vecteur de $T_{\xi} M^{A}$ tel que $\left(\varphi \circ \omega^{A}\right)(\xi)(X, Y)=0$ pour tout $Y \in T_{\xi} M^{A}$. On a donc

$$
\sum_{\substack{i=1,2, \ldots, 2 n \\ \alpha \in I}} \lambda_{i \alpha}\left(\varphi \circ \omega^{A}\right)(\xi)\left(a_{\alpha}\left(\frac{\partial}{\partial x_{i}}\right)^{A}(\xi), b\left(\frac{\partial}{\partial x_{j}}\right)^{A}(\xi)\right)=0
$$

pour tout $b \in A$ et pour tout $j=1,2, \cdots, 2 n$. On déduit que

$$
\sum_{\substack{i=1,2, \ldots, 2,2 \\ \alpha \in I ; \beta \in I}} \lambda_{i \alpha} \varphi\left(a_{\alpha} a_{\beta} b\right) a_{\beta}^{*}\left[\xi\left(\frac{\omega}{U}\left(\frac{\partial}{\partial x_{i}}, \frac{\partial}{\partial x_{j}}\right)\right)\right]=0 \text { pour tout } j=1,2, \cdots, 2 n \text {. }
$$

Pour $j=1,2, \cdots, n$ on a $\sum_{\alpha \in I} \lambda_{j+n} \varphi\left(a_{\alpha} b\right)=0$ pour tout $b \in A$. D'où $\sum_{\alpha \in I} \lambda_{j+n} a_{\alpha}$ est un élément de $\operatorname{ker}\left(\varphi \circ \mu_{A}\right)$. Pour $j=n+1, n+2, \cdots, 2 n$, $\sum_{\alpha \in I} \lambda_{i \alpha} a_{\alpha}$ est un élément de $\operatorname{ker}\left(\varphi \circ \mu_{A}\right)$ pour $i=1,2, \cdots, n$. On conclut donc que $\sum_{\alpha \in I} \lambda_{k_{\alpha}} a_{\alpha}$ appartient à $\operatorname{ker}\left(\varphi \circ \mu_{A}\right)$ pour $k=1,2, \cdots, 2 n$. On déduit donc que $X \in \operatorname{ker}\left(\varphi \circ \mu_{A}\right) \cdot T_{\xi} M^{A}$.

Inversement, soit $Z$ un élément de $\operatorname{ker}\left(\varphi \circ \mu_{A}\right) \cdot T_{\xi} M^{A}$. On écrit

$$
Z=\sum_{\sigma=\text { fini }} f_{\sigma} V_{\sigma} \text { où } f_{\sigma} \in \operatorname{ker}\left(\varphi \circ \mu_{A}\right) \text { et } V_{\sigma} \in T_{\xi} M^{A} \text {. }
$$

On a donc

$$
Z=\sum_{\substack{i=1,2, \ldots, 2,2 n \\ \alpha \in I ; \sigma=\text { fini }}} \lambda_{i \alpha}^{\sigma} f_{\sigma} a_{\alpha}\left(\frac{\partial}{\partial x_{i}}\right)^{A}(\xi)
$$

Pour tout $\beta$ dans $I$ et pour $j=1,2, \cdots, n$ alors

$$
\begin{aligned}
\left(\varphi \circ \omega^{A}\right)(\xi)\left(Z, a_{\beta}\left(\frac{\partial}{\partial x_{j}}\right)^{A}(\xi)\right) & =\sum_{\substack{i=1,2, \ldots, 2 n \\
\alpha, r \in I ; \sigma=\text { fini }}} \lambda_{i \alpha}^{\sigma} \varphi\left(f_{\sigma} a_{\alpha} a_{\beta} a_{r}^{*}\right) a_{r}^{*}\left[\xi\left(\omega / U\left(\frac{\partial}{\partial x_{i}}, \frac{\partial}{\partial x_{j}}\right)\right)\right] \\
& =\sum_{\substack{\alpha, \gamma \\
\alpha=\text { fini }}} \lambda_{j+n, \alpha}^{\sigma} \varphi\left(f_{\sigma} a_{\alpha} a_{\beta}\right) .
\end{aligned}
$$

Comme $f_{\alpha} \in \operatorname{ker}\left(\varphi \circ \mu_{A}\right)$, on a

$$
\left(\varphi \circ \omega^{A}\right)(\xi)\left(Z, a_{\beta}\left(\frac{\partial}{\partial x_{j}}\right)^{A}(\xi)\right)=0 \text { pour } j=1,2, \cdots, n .
$$

De la même façon, on a

$$
\left(\varphi \circ \omega^{A}\right)(\xi)\left(Z, a_{\beta}\left(\frac{\partial}{\partial x_{j}}\right)^{A}(\xi)\right)=0 \text { pour } j=n+1, n+2, \cdots, 2 n .
$$


On conclut que $\left(\varphi \circ \omega^{A}\right)(\xi)(Z, Y)=0$ pour tout $Y \in T_{\xi} M^{A}$. Etant donné un idéal $I$ de $A$, on a $\operatorname{dim}\left(I \cdot T_{\xi} M^{A}\right)=\operatorname{dim}(I) \times \operatorname{dim} M$ [6]. Comme $\operatorname{ker}\left(\varphi \circ \mu_{A}\right)$ est un idéal de $A$, alors $\operatorname{rang}\left(\varphi \circ \omega^{A}\right)=\operatorname{rang}\left(\varphi \circ \mu_{A}\right) \times \operatorname{dim} M$.

Lemme. Soit $\mathfrak{m}$ l'idéal de $A$ et ann(m) l'annulateur de $\mathfrak{m}$. Il existe une forme linéaire $\varphi$ sur A telle que la forme bilinéaire symétrique $\varphi \circ \mu_{A}$ : $A \times A \rightarrow \mathbb{R}$ soit non-dégénérée si et seulement si $\operatorname{dim}[\operatorname{ann}(\mathfrak{m})]=1$.

\section{Démonstration.}

Condition nécessaire. Désignons par $\pi: A \rightarrow \mathbb{R}$ l'augmentation. Soit $\varphi \in A^{*}$ une forme linéaire sur $A$ telle que $\varphi \circ \mu_{A}$ soit non-dégénérée et $a \in \operatorname{ann}(\mathfrak{m})$. Supposons $\varphi(a)=0$. Pour tout $b \in A, a b=a \cdot \pi(b)$. D'où $\varphi(a b)=\pi(b) \varphi(a)=0$ pour tout $b \in A$. Comme $\varphi \circ \mu_{A}$ est non-dégénérée alors $a=0$. Ainsi la restriction de $\varphi$ à $\operatorname{ann}(\mathfrak{m})$ est injective: d'où $\operatorname{dim}[\operatorname{ann}(\mathfrak{m})]$ $=1$.

Condition suffisante. On suppose $\operatorname{dim}[\operatorname{ann}(\mathfrak{m})]=1$. Soit $\varepsilon$ une base de $\operatorname{ann}(\mathfrak{m})$ et $\varphi \in A^{*}$ une forme linéaire sur $A$ telle que $\varphi[\operatorname{ann}(\mathfrak{m})] \neq 0$. On note $h$ la hauteur de $A:\left(\mathfrak{m}^{h} \neq(0)\right.$ et $\left.\mathfrak{m}^{h+1}=(0)\right)$. La suite croissante d'idéaux

$$
\mathfrak{m}^{h} \subset \mathfrak{m}^{h-1} \subset \mathfrak{m}^{h-2} \subset \cdots \subset \mathfrak{m}^{2} \subset \mathfrak{m}
$$

induit la suite décroissante

$$
\mathfrak{m}=\operatorname{ann}\left(\mathfrak{m}^{h}\right) \supset \operatorname{ann}\left(\mathfrak{m}^{h-1}\right) \supset \operatorname{ann}\left(\mathfrak{m}^{h-2}\right) \supset \cdots \supset \operatorname{ann}\left(\mathfrak{m}^{2}\right) \supset \operatorname{ann}(\mathfrak{m}) .
$$

Soit $a \in A$ tel que $\varphi(a b)=0$ pour tout $b$ dans $A$.

ler cas: $\quad b=\varepsilon$ base de ann(m).

On $a: 0=\varphi(a \varepsilon)=\varphi(\pi(a) \cdot \varepsilon)=\pi(a) \varphi(\varepsilon) . \quad$ Comme $\varphi(\varepsilon) \neq 0$, alors $\pi(a)=0$. Donc $a \in \mathfrak{m}$.

2e cas: $b \in \mathfrak{m}^{h-1}$ avec $a \in \mathfrak{m}$.

Ainsi $a b \in \mathfrak{m}^{h}=\operatorname{ann}(\mathfrak{m})$. On déduit que $a b=\lambda \varepsilon$. Comme $\varphi(a b)=0$, alors $a b=0$ pour tout $b \in \mathfrak{m}^{h-1}$. Donc $a \in \operatorname{ann}\left(\mathfrak{m}^{h-1}\right)$.

3e cas: $b \in \mathfrak{m}^{h-2}$ avec $a \in \operatorname{ann}\left(\mathfrak{m}^{h-1}\right)$.

Pour tout $c \in \mathfrak{m},(a b) c=a(b c)$. Puisque $b \in \mathfrak{m}^{h-2}$ et $c \in \mathfrak{m}$, alors $b c \in$ $\mathfrak{m}^{h-1}$. Comme $a \in \operatorname{ann}\left(\mathfrak{n t}^{h-1}\right)$, on conclut que $(a b) c=0$ pour tout $c \in \mathfrak{m}$; donc $a b \in \operatorname{ann}(\mathfrak{m})$. Ainsi, $a b=\lambda \varepsilon$ et $\varphi(a b)=0$ pour tout $b \in \mathfrak{m}^{h-2}$. On a 
donc $a b=0$ pour tout $b \in \mathfrak{m}^{h-2}$. D'où $a \in \operatorname{ann}\left(\mathfrak{m}^{h-2}\right)$.

Supposons $b \in \mathfrak{m}^{h-i-1}$ avec $a \in \operatorname{ann}\left(\mathfrak{m}^{h-i}\right)$. Pour tout $c \in \mathfrak{m},(a b) c=$ $a(b c)=0$. Ainsi $a b \in \operatorname{ann}(111)$. Comme $\varphi(a b)=0$ alors $a b=0$ pour tout $b \in \mathfrak{m}^{h-i-1}$. D'où $a \in \operatorname{ann}\left(\mathfrak{m}^{h-i-1}\right)$. Lorsque $i=h-2$, on a $a \in \operatorname{ann}(\mathfrak{m})$. Puisque $\varphi(a)=0$ alors $a=0$. La forme bilinéaire symétrique $\varphi \circ \mu_{A}: A \times A$ $\rightarrow \mathbb{R}$ est donc non-dégénérée.

Remarque. Lorsque $\operatorname{dim}[\operatorname{ann}(\mathfrak{m})]=1$, les seules formes $\varphi \in A^{*}$ telles $\varphi \circ \mu_{A}: A \times A \rightarrow \mathbb{R}$ soient non-dégénérées sont celles qui ne s'annulent pas $\operatorname{sur} \operatorname{ann}(\mathfrak{m})$.

EXEMPLE. Les algébres locales

$$
\mathbb{R}[T] /\left(T^{k}\right) ; \mathbb{R}\left[T_{1}, \cdots, T_{s}\right] /\left(T_{1}^{k_{1}}, T_{2}^{k_{2}}, \cdots, T_{s}^{k_{s}}\right)
$$

avec $k \geq 1, k_{1} \geq 1, \cdots, k_{s} \geq 1$ sont telles que $\operatorname{dim}[\operatorname{ann}(\mathfrak{m})]=1$.

Corollaire. Soit $(M, \omega)$ une variété symplectique. Etant donné une forme linéaire $\varphi \in A^{*}$, la forme scalaire $\varphi \circ \omega^{A}$ est une forme symplectique sur $M^{A}$ si et seulement si $\operatorname{dim}[\operatorname{ann}(\mathfrak{n t})]=1$ et $\varphi[\operatorname{ann}(\mathfrak{m})] \neq 0$.

Soit $I$ un idéal de A. La distribution $\xi \rightarrow I \cdot T_{\xi} M^{A}$ est un système différentiel de dimension $\operatorname{dim}(I) \times \operatorname{dim} M[6]$.

Proposition 3. Soit $(M, \omega)$ une variété symplectique. Etant donné $\varphi \in A^{*}$, l'orthogonal de $I \cdot T_{\xi} M^{A}$ par rapport à $\varphi \circ \omega^{A}$ est $I_{\varphi_{\circ} \mu_{A}}^{\perp} \cdot T_{\xi} M^{A}$ où $I_{\varphi_{\circ} \mu_{A}}^{\perp}$

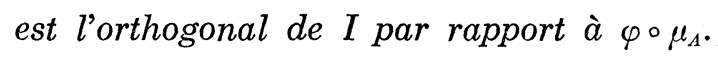

Démonstration. Soit $\xi$ un point proche de $x_{0}$ d'espèce $A,\left(x_{1}, \cdots, x_{2 n}\right)$ un système de coordonnées locales dans un voisinage $U$ de $x_{0}$ tel que

$$
\omega / U\left(\frac{\partial}{\partial x_{i}}, \frac{\partial}{\partial x_{i+n}}\right)=1 \text { pour } i=1,2, \cdots, n
$$

et

$$
\omega / U\left(\frac{\partial}{\partial x_{i}}, \frac{\partial}{\partial x_{j}}\right)=0 \text { pour } j \neq i+n
$$

Soit $X=\sum_{\substack{i=1,2, \ldots, 2 n \\ \alpha \in I}} \lambda_{i \alpha} a_{\alpha}\left(\partial / \partial x_{i}\right)^{A}(\xi)$ un vecteur de $T_{\xi} M^{A}$ qui appartient à l'orthogonal de ${ }^{\alpha \in I} \cdot T_{\xi} M^{A}$ par rapport à $\varphi \circ \omega^{A}$. Ainsi $\left(\varphi \circ \omega^{A}\right)(\xi)(X, Y)=0$ pour tout $Y \in I \cdot T_{\xi} M^{A}$. On a donc

$$
\sum_{\substack{i=1,2, \ldots, 2, n \\ \alpha \in I ; \beta \in I}} \lambda_{\alpha} \varphi\left(a_{\alpha} a_{\beta} b\right) a_{\beta}^{*}\left[\xi\left(\omega / U\left(\frac{\partial}{\partial x_{i}}, \frac{\partial}{\partial x_{j}}\right)\right)\right]=0 \text { pour tout } j=1,2, \cdots, 2 n .
$$


On vérifie que $\sum_{\alpha \in I} \lambda_{i \alpha} \varphi\left(a_{\alpha} b\right)=0$ pour tout $i=1,2, \cdots, 2 n$ et pour tout $b \in I$. On conclut que $\left(\varphi \circ \mu_{A}\right)\left(\sum_{\alpha \in I} \lambda_{i \alpha} a_{\alpha}, b\right)=0$ pour tout $b \in I$, c'est-à-dire que $\sum_{\alpha \in I} \lambda_{i \alpha} a_{\alpha}$ appartient à l'orthogonal de $I$ par rapport à $\varphi \circ \mu_{A}$. Le vecteur $X$ appartient ainsi à $I_{\varphi \circ \mu_{A}}^{\perp}, T_{\xi} M^{A}$. La réciproque se vérifie facilement.

Corollaire 1. Soit $(M, \omega)$ une variété symplectique. Si $A$ est une algèbre locale telle que $\operatorname{dim}[\operatorname{ann}(\mathfrak{m})]=1$, alors pour toute forme linéaire $\varphi \in A^{*}$ qui ne s'annule pas sur ann(m), l'orthogonal de $I \cdot T_{\xi} M^{A}$ par rapport $\grave{a} \varphi \circ \omega^{A}$ est $\operatorname{ann}(I) . T_{\xi} M^{A}$.

Corollaire 2. Soit $(M, \omega)$ une variété symplectique, $A$ une algèbre locale telle que $\operatorname{dim}[\operatorname{ann}(\mathfrak{m})]=1$ et $\varphi \in A^{*}$ une forme linéaire qui ne s'annule pas sur ann(m). Il n'existe pas sur $M^{A}$ de champ d'éléments de contact lagrangien de la forme $\xi \rightarrow I . T_{\xi} M^{A}$ pour $\varphi \circ \omega^{A}$ lorsque $A$ est de dimension impaire.

Démonstration. En effet, si $A$ est telle que $\operatorname{dimann}(\mathfrak{m})]=1$, on a: $\operatorname{dim} I+\operatorname{dim} \operatorname{ann}(I)=\operatorname{dim} A$ pour tout idéal $I$ de $A$. Si le champ d'éléments de contact $\xi \rightarrow I . T_{\xi} M^{A}$ est langrangien, alors $\operatorname{ann}(I)=I$. Ce qui implique que $\operatorname{dim} A=2 \operatorname{dim} I$. Ce qui signifie que $A$ est de dimension paire.

\section{§ 2. Relèvements des tenseurs symétriques de type $\left(\begin{array}{l}0 \\ 2\end{array}\right)$}

Proposition 4. Soit g: $\mathfrak{X}(M) \times \mathfrak{X}(M) \rightarrow C^{\infty}(M)$ un tenseur symétrique de type $\left(\begin{array}{l}0 \\ 2\end{array}\right)$ sur $M . \quad I l$ existe un tenseur symétrique de type $\left(\begin{array}{l}0 \\ 2\end{array}\right)$ et un seul $g^{A}$ sur $M^{A} \grave{a}$ valeurs dans A tel que: $g^{A}\left(a X^{A}, b Y^{A}\right)=a b[g(X, Y)]^{A}$ pour tous $a, b$ dans $A$ et $X, Y$ dans $\mathfrak{X}(M)$.

La démonstration se fait de la même façon que pour la proposition 1.

Si $\varphi \in A^{*}$ est une forme linéaire sur $A$, on dira que $\varphi \circ g^{A}$ est un relevé de $g$ à $M^{A}$.

Proposition 5. Soit $g$ un tenseur symétrique sur $M$ de type $\left(\begin{array}{l}0 \\ 2\end{array}\right)$ et de rang constant. Pour toute forme linéaire $\varphi$ sur $A$, on $a: \operatorname{rang}\left(\varphi \circ g^{A}\right)=$ $\operatorname{rang}\left(\varphi \circ \mu_{A}\right) \times \operatorname{rang}(g)$.

Ceci découle du lemme suivant:

LEMme. Soit $g$ un tenseur symétrique de type $\left(\begin{array}{l}0 \\ 2\end{array}\right)$ sur $M$, de rang constant et de signature $(p, q)$. Soit $\varphi \in A^{*}$ une forme linéaire sur $A$ et $(s, t)$ 
la signature de $\varphi \circ \mu_{A}$. Alors la signature de $\varphi \circ g^{A}$ est $(s p+t q, s q+t p)$.

Démonstration. Soit $\xi$ un point proche de $x_{0}$ et $g_{x_{0}}$ la forme bilinéaire symétrique sur $T_{x_{0}} M$ induite par $g$ et $\left(v_{1}, v_{2}, \cdots, v_{n}\right)$ une base orthogonale de $T_{x_{0}} M$ pour $g_{x_{0}}$. Soit $\left(a_{\alpha}\right)_{\alpha \in I}$ une base de $A$ orthogonale pour $\varphi \circ \mu_{A}$ et $\left(a_{\alpha}^{*}\right)_{\alpha \in I}$ la base duale. D'où:

$$
\begin{aligned}
\left(\varphi \circ g^{A}\right)(\xi)\left(a_{\alpha} v_{i}, a_{\beta} v_{j}\right) & =\sum_{r \in I} \varphi\left(a_{\alpha} a_{\beta} a_{r}\right) a_{r}^{*}\left[\xi\left(g_{x_{0}}\left(v_{i}, v_{j}\right)\right)\right] \\
& =0 \text { pour } i \neq j \text { ou } \alpha \neq \beta \\
\left(\varphi \circ g^{A}\right)(\xi)\left(a_{\alpha} v_{i}, a_{\alpha} v_{i}\right) & =\sum_{r \in I} \varphi\left(a_{\alpha}^{2} a_{r}\right) a_{r}^{*}\left[\xi\left(g_{x_{0}}\left(v_{i}, v_{i}\right)\right)\right] \\
& =\varphi\left(a_{\alpha}^{2}\right) g_{x_{0}}\left(v_{i}, v_{i}\right) \\
& =\left(\varphi \circ \mu_{A}\right)\left(a_{\alpha}, a_{\alpha}\right) g_{x_{0}}\left(v_{i}, v_{i}\right)
\end{aligned}
$$

Il s'ensuit que la signature de $\varphi \circ \mu_{A}$ est $(s p+t q, s q+t p)$.

CorollaIre. Soit $(M, g)$ une variété pseudo-riemannienne et $\varphi \in A^{*}$ une forme linéaire sur $A$. Le tenseur symétrique de type $\left(\begin{array}{l}0 \\ 2\end{array}\right), \varphi \circ g^{A}$, est une pseudo-métrique sur $M^{A}$ si et seulement si $\operatorname{dim}[\operatorname{ann}(\mathfrak{m})]=1$ et $\varphi[\operatorname{ann}(\mathfrak{m})] \neq 0$.

ExEmple. Soit $(M, g)$ une variété pseudo-riemannienne de dimension $n$. Soit $A=\mathbb{D}$, l'algèbre des nombres duaux, et $(1, \varepsilon)$ une base de $\mathbb{D}$ avec $\varepsilon^{2}=0,\left(1^{*}, \varepsilon^{*}\right)$ la base duale. Si $\left(x_{1}, \cdots, x_{n}\right)$ est un système de coordonnées locales de $M$ et $\left(y_{1}, \cdots, y_{n}\right)$ les coordonnées sur la fibre de $M^{\mathrm{D}}=T M$ et si $g\left(\partial / \partial x_{i}, \partial / \partial x_{j}\right)=g_{i, j}$, alors

$$
\varepsilon^{*} \circ g^{\mathrm{D}}=\left(\begin{array}{c|c}
\sum_{k=1}^{n} y_{k} \frac{\partial g_{i j}}{\partial x_{k}} & g_{i j} \\
\hline g_{i j} & O
\end{array}\right) .
$$

\section{Remarques.}

1) La relevée d'une métrique n'est jamais une métrique.

2) Si $(M, g)$ est une variété pseudo-riemannienne, pour toute forme linéaire $\varphi \in \mathbb{D}^{*}$ qui ne s'annule pas sur l'idéal maximal de $\mathbb{D}$, la signature de $\varphi \circ g^{D}$ ne dépend pas de la singature de $g$.

Proposition 6 [4]. Etant donné une connexion linéaire $\nabla$ sur $M$, il existe une connexion linéaire $\nabla^{A}$ et une seule sur $M^{A}$ telle que: $\nabla_{a X^{A}}^{A} b Y^{A}$ $=a b\left(\nabla_{X} Y\right)^{A}$ pour tous $a, b$ dans $A$ et $X, Y$ dans $\mathfrak{X}(M)$.

Proposition 7. Soit $\nabla$ une connexion linéaire sur $M$. Pour tout 
tenseur symétrique $g$ de type $\left(\begin{array}{l}0 \\ 2\end{array}\right)$ sur $M$, on $a: \nabla^{A} g^{A}=(\nabla g)^{A}$. De plus, $\nabla^{A}\left(\varphi \circ g^{A}\right)=\varphi \circ(\nabla g)^{A}$ pour toute forme linéaire $\varphi$ sur $A$.

La démonstration ne présente aucune difficulté.

Corollaire. Soit $(M, g)$ une variété pseudo-riemannienne et $\nabla_{g}$ la connexion linéaire sur $M$ déduite de $g$ Si $A$ est une algèbre locale telle que $\operatorname{dim}[\operatorname{ann}(\mathfrak{m})]=1$, alors pour toute forme linéaire $\varphi \in A^{*}$ qui ne s'annule pas sur ann $(\mathfrak{m}),\left(\nabla_{g}\right)^{A}$ est la connexion linéaire sur $M^{A}$ déduite de $\varphi \circ g^{A}$.

Remarque. Soit $\operatorname{ann}(\mathfrak{m})^{\perp}$ l'espace des formes lineaires sur $A$ qui s'annulent sur ann $(\mathfrak{m})$ et $\mathbb{P}\left(A^{*} / \operatorname{ann}(\mathfrak{m})^{\perp}\right)$ l'espace projectif de $A^{*} / \operatorname{ann}(\mathfrak{m})^{\perp}$. Soit $(M, \omega)$ une variété symplectique (respectivement soit $(M, g)$ une variété pseudo-riemannienne). Si $\operatorname{dim}[\operatorname{ann}(\mathfrak{m})]=1$ et si $\varphi \in A^{*}$, le fait que $\varphi \circ \omega^{A}$ ou $\varphi \circ g^{A}$ soit non-dégénérée ne dépend pas de $\varphi$ mais de la classe de $\varphi$ dans $\mathbb{P}\left(A^{*} / \operatorname{ann}(\mathfrak{m})^{\perp}\right)$. De même si $\varphi \circ g^{A}$ est une pseudo-métrique sur $M^{A}$, la connexion linéaire sur $M^{A}$ déduite de $\varphi \circ g^{A}$ ne dépend pas de $\varphi$ mais de la classe de $\varphi$ dans $\mathbb{P}\left(A^{*} / \operatorname{ann}(\mathfrak{m})^{\perp}\right)$.

\section{BIBILIOGRAHPIE}

[1] Morimoto, A., Prolongations of $G$-structures to tangent bundles of higher order, Nagoya Math. J., 38 (1970), 153-179.

[2] - Liftings of some types of tensor fields and connections to tangent bundles of $p^{r}$-velocities, Nagoya Math. J., 40 (1970), 13-31.

[ 3 ] - Liftings of tensor fields and connections to tangent bundles of higher order, Nagoya Math. J., 40 (1970), 99-120.

[4] - Prolongation of connections to bundles of infinitely near points, J. Diff. Geom., 11 (1976), 479-498.

[5] Okassa, E., Prolongement des champs de vecteurs à des variétés de points proches, C. R. Acad. Sci. Paris, série I Math. t. 300, 6 (1985), 173-176.

[6] - Prolongement des champs de vecteurs à des variétés de points proches, Prépublication de l'Institut Fourier, Grenoble, 1987.

[ 7 ] Yano, K. and Ishihara, S., Tangent and cotangent bundles, Diff. Geom. Marcel Dekker, New-York, 1973.

[ 8 ] Yano, K. and Patterson, E. M., Vertical and complete lifts from a manifold to its cotangent bundles, Jour. Math. Soc. Japan, 19 (1967), 91-113.

[9] Weil, A., Théorie des points proches sur les variétés différentiables, Colloque Geom. Diff. Strasbourg, 111-117, 1953.

Université de Grenoble I

Institut Fourier

Laboratoire de Mathématiques

B.P. 74

38402 ST-MARTIN-D'HÈRES (France) 
Université Marien Ngouabi

Faculté des Sciences

Département de Mathématiques

B.P. 69

BRAZZAVILLE (Congo) 